Valbenazine is a new FDA-approved treatment for adults with tardive dyskinesia, representing a further avenue for management. Greater focus on patient involvement, and communication surrounding anticipated side effects, is likely to benefit compliance with treatment and improve the doctor-patient relationship.

\section{Audit on the documentation of ethnicity within CAMHS}

Charlotte Scott* and Roger Lakin

Tees, Esk and Wear Valleys Foundation NHS Trust

${ }^{\star}$ Corresponding author.

doi: 10.1192/bjo.2021.304

Aims. Evidence suggests children from minority ethnic groups have lower rates of referrals from primary care to CAMHS, are more likely to be referred via involuntary or compulsory routes and less likely to have access to therapies than children from white backgrounds. In order to understand how ethnicity influences individuals and ensure service innovation meet these needs data collected have to be accurate. The Mental Health Services Data Set outlines all children and families receiving NHS care should have ethnicity included as a mandatory data submission and services are performance managed on this.

The aim of this audit to review the documentation of ethnicity for service users in CAMHS. We agreed that 100\% of patients within York and North Yorkshire (Y\&NY) CAMHS should have their ethnicity documented.

Method. Integrated Information Centre (IIC) was used to collect data on the documentation of ethnicity for patients under Y\&NY CAMHS on 27th August 2020.

Result. The total caseload was 4109 patients.

$823(20 \%)$ had their ethnicity documented as 'unknown' (the clinician had entered 'unknown' or the patient has 'declined to disclose').

49 (1.2\%) patients had no entry regarding ethnicity (missing). Conclusion. We recommend further exploration to consider why 1 in 5 patients have 'unknown ethnicity' documented. We recommend: conducting a refined search considering the percentage of 'declined to disclose' and 'not stated' within the 'unknown ethnicity' section emailing care coordinators for patients with 'unknown ethnicity' or 'missing ethnicity' conducting a questionnaire to gather the opinions and experiences of clinicians, patients and families when talking about ethnicity

reviewing the process for documenting ethnicity to improve accuracy developing staff training, to promote a culture of confidence and curiosity when discussing ethnicity Following this intervention we will aim to re-audit and consider if this has improved the rates of documentation of ethnicity.

\section{COVID-19 antibody seroprevalence in residential psychiatric inpatients}

Sheena Shah ${ }^{1 \star}$, Arshad Hussain ${ }^{1}$, Sabreena Qadri ${ }^{1}$, Fazle Roub ${ }^{1}$, Insha Rauf ${ }^{1}$ and Praveen Kumar ${ }^{2}$

${ }^{1}$ Institute of Mental Health and Neurosciences and ${ }^{2}$ NHS Scotland ${ }^{\star}$ Corresponding author.

doi: 10.1192/bjo.2021.305

Aims. While other mental health care outpatient facilities were moved to COVID-centers in March 2020 during the COVID-19 pandemic, the Institute of Mental Health and Neurosciences in Kashmir remained the only functional outpatient facility in the region. It is the only mental health care hospital in the country with a residential facility for psychiatric inpatients catering to the whole population of Jammu and Kashmir, India. The Mental Health Care Act 2017 that neccesitated "halfway homes" is yet to be implemented in the state leaving it's inpatients entirely under the institution's care. This study is to investigate the seroprevalence of antibodies to SARS-COVID-19 virus in the 34 residential inpatients in separate male (23 patients) and female (11 patients) wards. This was done as an audit to strategies and measures taken by the institute in protecting it's inpatients.

Method. 3 to $5 \mathrm{ml}$ of peripheral venous blood samples were collected and plasma extracted and analysed using the CE-IVD Roche Cobas Elecsys AntiSARS-CoV-2, Electrochemiluminescence Immunoassay (ECLIA) for the qualitative detection of total Immunoglobulins (IgG, IgM and IgA; Pan Ig) generated against SARS-CoV-2 (Roche Diagnostics, Indianapolis, IN, USA). The test was performed according to the manufacturer's instructions.

Result. Out of the 34 inpatients, 2 male inpatients tested positive for antibodies against SARS-CoV-2 (seroprevalence of 5.88\%). In comparison, based on a report conducted by the government's Department of Community Medicine and Biochemistry on the 28 th of October 2020, out of 2,361 participants in the community, 959 tested positive (seroprevalence of $40.6 \%$ ).

One of the inpatients that tested positive was re-admitted after testing negative via RT-PCR. The second patient was admitted after being found homeless. He was tested negative on day 1 via RAT and on day 5 via RT-PCR. We believe both of them aquired the infection in the community.

Conclusion. This audit shows that the strategies implemented by the institute were effective in the prevention of the spread of COVID-19. Practical implementations of what works and improvisations are the proven methods of decreasing the mortality and morbidity in vulnerable populations while continuously providing vital mental health services.

An assessment of referrals to a liaison psychiatry team within a large district general hospital - Completing the Cycle

Ivan Shanley*, Sophie Tillman, Shruti Lodhi and Shazia Shabbir Surrey and Borders NHS Foundation Trust, Frimley Park Hospital ${ }^{\star}$ Corresponding author.

doi: 10.1192/bjo.2021.306

Aims. In 2019 members of the Liaison Psychiatry Department at Frimley Park Hospital completed an audit of the referrals to the service1. The quality of referrals was found to be highly variable, for example only $28 \%$ included a risk assessment and frequently omitted both past psychiatric and past medical histories. As such an intervention was designed involving three parts;

Multidisciplinary education of staff

New and more readily available referral guidelines

New referral form

This re-audit seeks to complete the audit cycle and assess the impact of the intervention.

Method. The first 50 referrals to the Liaison Psychiatry Department of Frimley Park Hospital during February 2021 were assessed using the following criteria:

Staff type, referral source, physically fit for assessment, physical cause ruled out, drugs / alcohol involved, appropriate reason for referral, clinical question asked, did final diagnosis match referral diagnosis, risk assessment included, information about admission included, past psychiatric history included and past medical history included. 